\title{
CIENCIAMATRIA
}

Revista Interdisciplinaria de Humanidades, Educación, Ciencia y Tecnología

Año VII. Vol. VII. N¹2. Enero - Junio. 2021

Hecho el depósito de ley: pp201602FA4721

ISSN-L: 2542-3029; ISSN: 2610-802X

Universidad Nacional Experimental Francisco de Miranda (UNEFM). Santa Ana de Coro. Venezuela

Alexandra Kimberly Tamay-Chimborazo; Juan Carlos Erazo-Álvarez; Cecilia Ivonne Narváez-Zurita; Cristián Andrés Erazo-Álvarez

DOI $10.35381 / \mathrm{cm} \cdot \mathrm{v} 7 \mathrm{i} 12.453$

\section{Marketing experiencial como estrategia de generación de ingresos}

\section{Experiential marketing as a revenue generation}

Alexandra Kimberly Tamay-Chimborazo alexandra.tamay.77@est.ucacue.edu.ec Universidad Católica de Cuenca, Cuenca

Ecuador

https://orcid.org/0000-0002-6146-8446

Juan Carlos Erazo-Álvarez jcerazo@ucacue.edu.ec

Universidad Católica de Cuenca, Cuenca

Ecuador

https://orcid.org/0000-0001-6480-2270

Cecilia Ivonne Narváez-Zurita inarvaez@ucacue.edu.ec

Universidad Católica de Cuenca, Cuenca

Ecuador

https://orcid.org/0000-0002-7437-9880

Cristián Andrés Erazo-Álvarez

cristianerazo@ucacue.edu.ec

Universidad Católica de Cuenca, Cuenca

Ecuador

https://orcid.org/0000-0001-8746-4788

Recibido: 01 de octubre de 2020

Aprobado: 15 de diciembre de 2020 
Revista Interdisciplinaria de Humanidades, Educación, Ciencia y Tecnología

Año VII. Vol. VII. N¹2. Enero - Junio. 2021

Hecho el depósito de ley: pp201602FA4721

ISSN-L: 2542-3029; ISSN: 2610-802X

Universidad Nacional Experimental Francisco de Miranda (UNEFM). Santa Ana de Coro. Venezuela

Alexandra Kimberly Tamay-Chimborazo; Juan Carlos Erazo-Álvarez; Cecilia Ivonne Narváez-Zurita; Cristián Andrés Erazo-Álvarez

\title{
RESUMEN
}

El objetivo de la investigación se basó en diseñar estrategias de marketing experiencial como estrategia de generación de ingresos en el Motel San Valentín, ubicado en Azogues - Ecuador. El alcance metodológico empleado ha sido descriptivo transversal no experimental, la muestra poblacional fue de 34 personas al azar, en edades comprendidas entre los 18 y 65 años de edad, considerados potenciales usuarios del hotel objeto de estudio. En el desarrollo de la investigación se sugiere que el marketing experiencial busca generar una conexión emocional entre la marca y el comprador que los lleve a convertirse en fieles entusiastas de la marca, dispuestos a compartir su experiencia con otras personas dentro del motel, para lo cual se utilizarán diversas tácticas apelando a todos los sentidos probables que provoquen una inmersión emocional del comprador.

Descriptores: Marketing; afectividad; comercio; competencia económica. (Palabras tomadas del Tesauro UNESCO).

\begin{abstract}
The objective of the research was based on designing experiential marketing strategies as an income generation strategy at the San Valentín Motel, located in Azogues Ecuador. The methodological scope used has been descriptive, non-experimental, crosssectional, the population sample was 34 people at random, aged between 18 and 65 years old, considered potential users of the hotel under study. In the development of the research, it is suggested that experiential marketing seeks to generate an emotional connection between the brand and the buyer that leads them to become loyal fans of the brand, willing to share their experience with other people within the motel, for which various tactics will be used appealing to all the probable senses that provoke an emotional immersion of the buyer.
\end{abstract}

Descriptors: Marketing; emotions; trade; economic competition. (Words taken from the UNESCO Thesaurus). 


\section{CIENCIAMATRIA}

Revista Interdisciplinaria de Humanidades, Educación, Ciencia y Tecnología

Año VII. Vol. VII. N¹2. Enero - Junio. 2021

Hecho el depósito de ley: pp201602FA4721

ISSN-L: 2542-3029; ISSN: 2610-802X

Universidad Nacional Experimental Francisco de Miranda (UNEFM). Santa Ana de Coro. Venezuela

Alexandra Kimberly Tamay-Chimborazo; Juan Carlos Erazo-Álvarez; Cecilia Ivonne Narváez-Zurita; Cristián Andrés Erazo-Álvarez

\section{INTRODUCCIÓN}

El marketing experiencial se caracteriza porque provoca sensaciones que quedan en la memoria del cliente, gracias a la utilización de una gama amplia de herramientas que generan este vínculo, dichas herramientas han ido evolucionando en el tiempo, siempre con el objetivo de generar satisfacción en el cliente y lograr el cumplimiento de los objetivos organizacionales (Moreno, 2007). La funcionalidad del marketing en la economía es la de acomodar el trueque voluntario y competitivo de forma que asegure un encuentro eficiente entre la oferta y la demanda de productos y servicios, beneficiando al consumidor. De forma tradicional, las empresas han utilizado al marketing para llevar a cabo estudios de mercado, partiendo con la premisa que los consumidores adquieren compras racionales, es decir, tomando en cuenta la calidad y su comparación con el precio. En la actualidad, los clientes toman en cuenta cierto atributos que los diferencian de los competidores para decidir la compra (Caridad, et al., 2015).

Existe una conexión entre los sentidos y la adquisición de los bienes y servicios, en este contexto, la vista es el sentido que mayor influencia tiene en el marketing, la elección de formas y colores, el diseño de los aspectos de comercialización, la ejecución de campañas de publicitarias, son componentes que determinan el triunfo o decepción de las organizaciones (Barrios, 2012). Desde sus inicios, el sector de moteles, se aplicó técnicas de marketing tradicional, donde primaba la calidad del servicio, hoy en día, se pretende brindar experiencias memorables que den como resultado el aumento de la participación de mercado de este sector económico. El Motel San Valentín, ubicado en la ciudad de Biblián - Cañar, es una empresa dedicada al brindar servicios de alojamiento temporal, por medio de la modalidad de motel, con una trayectoria de 11 años, desde su inauguración se utilizó técnicas de marketing tradicional.

El objetivo de la investigación se basó en diseñar estrategias de marketing experiencial como estrategia de generación de ingresos en el Motel San Valentín, ubicado en Azogues - Ecuador. 


\section{CIENCIAMATRIA}

Revista Interdisciplinaria de Humanidades, Educación, Ciencia y Tecnología

Año VII. Vol. VII. N¹2. Enero - Junio. 2021

Hecho el depósito de ley: pp201602FA4721

ISSN-L: 2542-3029; ISSN: 2610-802X

Universidad Nacional Experimental Francisco de Miranda (UNEFM). Santa Ana de Coro. Venezuela

Alexandra Kimberly Tamay-Chimborazo; Juan Carlos Erazo-Álvarez; Cecilia Ivonne Narváez-Zurita;

Cristián Andrés Erazo-Álvarez

\section{Referencial teórico}

\section{Conceptos de marketing tradicional y experiencial}

Reflexionando sobre el recorrido que ha tenido el marketing experiencial en una herramienta sustancial, dentro del trabajo a la hora de prestar un servicio se logrará que el cliente tenga un hábito emocional que satisfaga sus insuficiencias. El concepto de comercialización tiene dos significados: En el primero, la comercialización se refiere al negocio, la logística y la seducción del comprador; mientras que, en el segundo, la comercialización tiene por objeto satisfacer las insuficiencias de los individuos y forjar cambios en ellos en absoluto a través de la trascendencia de las ocupaciones culturales, educativas y sanitarias, tratando el hábito competente de los recursos utilizables (OliveraCarhuaz \& Pulido-Capurro, 2018).

Complementando la propuesta anterior, el Marketing tenía inicialmente dos planes orientados a la sociedad, claramente definidos de la siguiente manera: Un área consistía en la distribución de la riqueza y la mediación, en la que la otra buscaba explicar de alguna manera el cambio racional de los mercados y sistemas, por lo que se garantiza que la comercialización no sólo cuida los intereses empresariales, sino también de la sociedad en su conjunto (Suárez del Villar \& Salazar Caballero, 2016).

Siguiendo estas definiciones, tenemos la posibilidad de resaltar que en el marketing experiencial el comprador escogerá el producto o servicio en funcionalidad de la experiencia que le da anteriormente y a lo largo del consumo, y no por exteriores del producto. Por consiguiente, el propósito primordial de la tendencia es concentrarse en el comprador y en las vivencias que se recibe previamente y a lo largo de la utilización y venta del bien o servicio (Lobera, 2018).

Hay 3 argumentos relevantes para describir el cambio progresivo del marketing clásico al marketing experiencial o de vivencias. En el marketing experimental, el neuromarketing es esencial ya que nos permite entender la mente del comprador para conocer sus 


\section{CIENCIAMATRIA}

Revista Interdisciplinaria de Humanidades, Educación, Ciencia y Tecnología

Año VII. Vol. VII. N¹2. Enero - Junio. 2021

Hecho el depósito de ley: pp201602FA4721

ISSN-L: 2542-3029; ISSN: 2610-802X

Universidad Nacional Experimental Francisco de Miranda (UNEFM). Santa Ana de Coro. Venezuela

Alexandra Kimberly Tamay-Chimborazo; Juan Carlos Erazo-Álvarez; Cecilia Ivonne Narváez-Zurita; Cristián Andrés Erazo-Álvarez

emociones y de esta manera llamar la atención sobre el servicio que queremos vender (Schimitt, 2000).

La marca y la relación del producto: Aproximadamente desde el principio, las marcas han determinado una continua reciprocidad con los bienes; Este parentesco es proporcionado por elementos de beneficio que ofrecen custodia, contradicción y libertad a los bienes en relación con otros (Cepeda-Palacio, 2014). La compra de productos de menor calidad, la competencia traicionera o los efectos externos nocivos que pueden encontrar en el mercado y que suponen una amenaza para el consumo y las buenas prácticas comerciales (Klotler \& Armstrong, 2008); aquí se destaca la defensa como una de las principales funciones de los logotipos, ya que permite al consumidor diferenciar un bien de otro, protegiéndolo de productos imperfectos o de atributos inferiores y favoreciendo la custodia de un determinado producto tal como lo proporciona la marca.

La calidad del servicio y la satisfacción del comprador, tienen la posibilidad de mejorar y tratar los intereses de las particularidades de los servicios; como no todas las particularidades tienen el mismo papel, es esencial encontrar el efecto de su manejo (Mejías-Acosta, et al., 2018). La autocomplacencia del comprador se convierte en un objetivo fundamental para cualquier organización que busque una interacción a largo plazo con el comprador. Esto se considera una prioridad máxima para la organización. Muchas empresas han reconocido la necesidad de perfeccionar sus estrategias con productos relacionados con las características de los mismos y examinar qué componentes indican el gusto del comprador.

\section{Evolución marketing tradicional al marketing experiencial}

El marketing ha experimentado una gigantesca evolución durante su tradición. Ha ocurrido de ofrecer solo los productos a ofrecer una vivencia o un disfrute (Ordónez \& Prieto, 2019). El marketing clásico ha degenerado su capacidad de atrapar al cliente, por el momento no vale la pena delimitar la comercialización de un producto afirma la 
usabilidad en la vinculación del cliente a través de sensaciones, sentimientos y emociones. Lo fundamental en la elección de la adquisición de un producto es la sensación; la experiencia que el cliente aprecia se cambia como una estrategia de venta que implica un coste añadido; la innovación de un producto en una experiencia.

El cliente actual da su opinión sobre sus experiencias para utilizarlas como parte de su tiempo de diversión o sus propias y generales recomendaciones $\mathrm{O}$ la adquisición no es la compra de tal producto, sino una experiencia que influye en otros conocimientos, el comprador se pregunta cuál es el producto adecuado para cada momento (Suárez del Villar \& Salazar Caballero, 2016).

\section{Tabla 1.}

Marketing Tradicional vs. Marketing Experiencial.

\begin{tabular}{ll}
\hline \multicolumn{1}{c}{ Marketing tradicional } & \multicolumn{1}{c}{ Marketing Experiencial } \\
\hline $\begin{array}{l}\text { Se reúne en las negocios particulares } \\
\text { Resalta las características del producto }\end{array}$ & $\begin{array}{l}\text { Se concentra en la retención del cliente } \\
\text { saciar al cliente }\end{array}$ \\
Plantea una visión a corto plazo & Plantea una aspecto a amplio plazo \\
Existe un bajo nivel de responsabilidad & Existe un eminente nivel de \\
con el cliente & responsabilidad con el cliente \\
Existe un nivel moderado de contacto con & Existe un elevado grado de relación con el \\
el cliente & cliente \\
La calidad se concentra, casi & La calidad corresponde a toda la empresa \\
exclusivamente en el producto &
\end{tabular}

Fuente: Kuster, et al. (2009). 


\section{CIENCIAMATRIA}

Revista Interdisciplinaria de Humanidades, Educación, Ciencia y Tecnología

Año VII. Vol. VII. N¹2. Enero - Junio. 2021

Hecho el depósito de ley: pp201602FA4721

ISSN-L: 2542-3029; ISSN: 2610-802X

Universidad Nacional Experimental Francisco de Miranda (UNEFM). Santa Ana de Coro. Venezuela

Alexandra Kimberly Tamay-Chimborazo; Juan Carlos Erazo-Álvarez; Cecilia Ivonne Narváez-Zurita; Cristián Andrés Erazo-Álvarez

\section{Neuromarketing dentro del marketing}

Hoy en día ni solo se busca satisfacer las necesidades de los clientes, sino darle un valor agregado al servicio pueda tener una experiencia diferente, puesto que principalmente es brindar el servicio que desea y conoce las necesidades mediante el cual se puede sentir satisfecho y tener una experiencia que cumpla con todas las expectativas que tienen, ya que ni se trata solo de vender un servicio, sino de vender una experiencia o algo fuera de lo común saliendo de lo tradicional, siempre viendo lo que desea el cliente. Neuromarketing es la exploración de la mente y el cerebro por varios años ha creado monumentales retos, algunas disciplinas han venido afrontando el argumento, como la doctrina, personalidad o el procedimiento y la integración de las neurociencias en los últimos años (Salas-Canales, 2018).

Sin apropiación, a pesar del tremendo progreso tecnológico, todavía has varias preguntas sin respuesta desde la antigüedad, incluyendo ¿dónde existe la conciencia?, ¿qué es la felicidad?, ¿cómo recordamos?, o simplemente ¿por qué elegimos determinada marca, producto o servicio? Ni resulta nada factible establecer aspectos como la atención, la memoria o el sentimiento, conocer el cerebro y la mente constituye un verdadero desafío. Es decir, las emociones, sentimientos y experiencias están ligadas directamente con la mente, ya que está nos ayuda a reflejar lo que cada persona piensa o siente.

\section{El marketing emocional y sensorial a través de los estímulos}

El marketing sensorial aporta una nueva dimensión a las empresas a través de la cual pueden interactuar con los consumidores y las audiencias (Bernal \& Galvis, 2008). Cada sentimiento podría ser impulsado a construir una imagen de marca más intensa y sostenible que afecte inmediatamente a la percepción del atributo del producto, incluido su coste. Esta es una de las pocas maneras de asegurar una verdadera mejora profesional. Martin Lindstrom aseguró que las empresas que se centran en el olfato, el gusto y el tacto, además de la vista y el oído, pueden lograr efectos psicológicos que 


\section{CIENCIAMATRIA}

Revista Interdisciplinaria de Humanidades, Educación, Ciencia y Tecnología

Año VII. Vol. VII. N¹2. Enero - Junio. 2021

Hecho el depósito de ley: pp201602FA4721

ISSN-L: 2542-3029; ISSN: 2610-802X

Universidad Nacional Experimental Francisco de Miranda (UNEFM). Santa Ana de Coro. Venezuela

Alexandra Kimberly Tamay-Chimborazo; Juan Carlos Erazo-Álvarez; Cecilia Ivonne Narváez-Zurita; Cristián Andrés Erazo-Álvarez

ayudan a duplicar la conciencia de marca y fortalecer la imagen y el posicionamiento de ésta sobre la suya a lo largo del camino.

Se establece que el más grande auge se proporcionó durante el siglo XXI una vez que se produjo el mayor desarrollo de indagaciones en neurociencia (De-Garcillán-LópezRúa, et al., 2015). El enfoque del marketing sensorial trata de compensar las deficiencias del marketing clásico, que es bastante racional. Además, se centra en las prácticas vividas por los clientes y sus sentimientos en este proceso. El propósito es que el consumidor se porte según los impulsos y emociones, que sobre el conocimiento. E marketing tradicional se apoya en la iniciativa del consumidor es racional y de que su comportamiento puede descomponerse en una secuencia de pasos realmente bien definidos y razonados, según la oferta que existe, la competencia, la satisfacción de las necesidades, etcétera. El marketing sensorial, por otro lado, colocan las vivencias y las emociones vividas y experimentadas por los clientes en el centro del proceso. Lo cual mueve a los humanos es el sentimiento, no el motivo (Reyes, et al., 2020).

La mayor parte de los individuos demuestran su lado emocional y las vivencias vividas diariamente sin que importe, dejando de lado el motivo. El Marketing Sensorial quiere crear una experiencia de compra exclusiva y única (De-Garcillán-López-Rúa, et al., 2015). Degustar, olfatear, notar, escuchar a qué suena y sentiremos cómo se aprecia el producto, es resultado de una estrategia dirigido a la comprensión completa del cliente, encaminada a manipular todas las fibras cuantas sean posibles y atrapar todo su cuerpo. La iniciativa debe atraer a los consumidores por medio de estímulos como recursos auditivos, olfativos, gustativos, entre otros. El propósito es que el individuo se vea inducida a probar el producto o servicio que se está promocionando, para que lo compre. El marketing sensorial se basa en un plan que se reúne en reforzar los sentimientos o sensaciones de los clientes fundamentalmente (Calle-Calderón, et al., 2020).

El marketing de las sensaciones y el marketing de los sentimientos a través de cierto marketing emocional; el marketing de las sensaciones se entiende como aquel cuyo 


\section{CIENCIAMATRIA}

Revista Interdisciplinaria de Humanidades, Educación, Ciencia y Tecnología

Año VII. Vol. VII. N¹2. Enero - Junio. 2021

Hecho el depósito de ley: pp201602FA4721

ISSN-L: 2542-3029; ISSN: 2610-802X

Universidad Nacional Experimental Francisco de Miranda (UNEFM). Santa Ana de Coro. Venezuela

Alexandra Kimberly Tamay-Chimborazo; Juan Carlos Erazo-Álvarez; Cecilia Ivonne Narváez-Zurita; Cristián Andrés Erazo-Álvarez

propósito es proporcionar placer estético, emociones, belleza y satisfacción a través de la estimulación sensorial. Para lograr un efecto sensorial, se define el modelo EPC (estímulo, proceso y consecuencia). El estímulo es la elección. prestando atención a la información recibida y manteniendo o almacenando la búsqueda sensorial para hacer más efectivos los estímulos relacionados con situaciones vividas o significativas (LazoTorres, et al., 2019).

El proceso depende de cómo se lleve a cabo el estímulo, que son diferentes modalidades de transmisión de la información (visual, auditiva, olfativa y táctil). En este contexto, el marketing sensorial o de los sentidos se forma como un elemento de diferenciación por parte del cliente a través de la motivación de los sentidos se atrae (Ramírez, Duarte, \& Toro, 2016). Dentro del marketing sensorial consideramos los siguientes estímulos como se muestra en la siguiente figura con la cual se atrae al cliente.
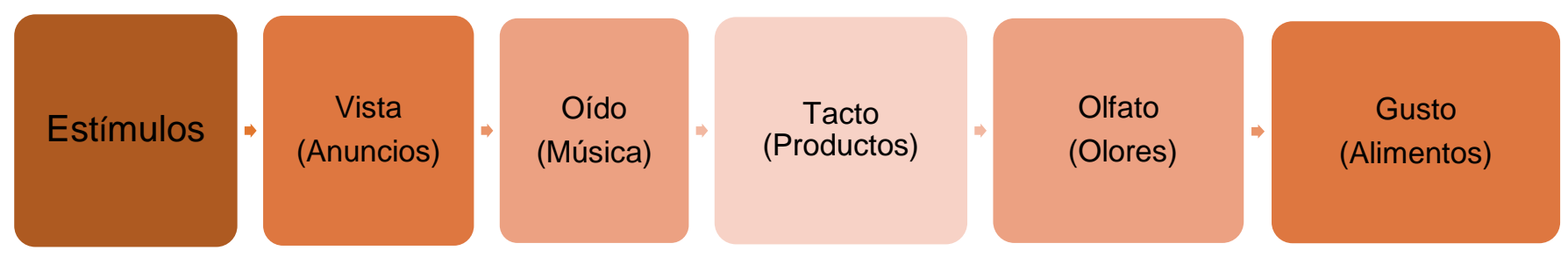

Figura 1. Estímulos.

Fuente: Elaboración propia. 


\section{CIENCIAMATRIA}

Revista Interdisciplinaria de Humanidades, Educación, Ciencia y Tecnología

Año VII. Vol. VII. N¹2. Enero - Junio. 2021

Hecho el depósito de ley: pp201602FA4721

ISSN-L: 2542-3029; ISSN: 2610-802X

Universidad Nacional Experimental Francisco de Miranda (UNEFM). Santa Ana de Coro. Venezuela

Alexandra Kimberly Tamay-Chimborazo; Juan Carlos Erazo-Álvarez; Cecilia Ivonne Narváez-Zurita; Cristián Andrés Erazo-Álvarez

\section{Satisfacción del comprador al adquirir un servicio}

El marketing emocional es fundamental para ofrecer coste y producir lealtad del cliente, lo cual es necesario para los beneficios, el crecimiento y los éxitos empresariales a largo plazo. Iniciemos definiendo la emoción en este ámbito. La confianza es la emoción de la compañía. Para tener un alto nivel de confianza, es importante que toda la empresa sea dirigida soberanamente en las estrategias en las que ella, como sus clientes, gane, tal vez experimente por sí misma cómo su corazón le lleva a hacer una elección en lugar de lo que dice su cabeza. En realidad, usted sabe que la emoción es la que marca la diferencia. Así pues ¿cómo explicar este misterioso paradigma? Piense en las elecciones de compra se basan en la emoción. Es cierto que hay un pensamiento racional, pero está influenciado por las emociones de una manera u otra (Cadavid-Gómez, 2004).

Es importante saber acerca de los choques en los que la atención conduce, ya que estos están socialmente desarrollados y construidos. El costo de saber cómo se generan las emociones radica en que juegan un papel crítico en importantes eventos humanos. De manera sintética, definen las emociones como una "parte integral e indispensable de la organización diaria de la vida"; como cualquiera de los sentimientos que uno tiene en un día: tristeza, alegría, compromiso, miedo, etc. Desde una perspectiva integradora, definen la emoción como "un conjunto complejo entre elementos subjetivos y objetivos, influenciado por los sistemas neuronales $u$ hormonales que pueden crear: a) experiencias afectivas como sentimientos de aceleración, gustos o disgustos, b) procesos cognitivos como perspicacia y evaluaciones, c) activación de ajustes fisiológicos, y d) comportamiento que es principalmente, pero no una y otra vez, expresivo, dirigido a un objetivo, y adaptativo (Carbache-Mora, et al., 2019).

La satisfacción se determina mediante la evaluación posterior de la calidad del servicio prestado en comparación con las modalidades anteriores. La satisfacción del cliente se logra cuando las expectativas del cliente se cumplen con servicios de alta calidad. Para satisfacer a los clientes es necesario conocer sus expectativas, ofrecer un servicio 


\section{CIENCIAMATRIA}

Revista Interdisciplinaria de Humanidades, Educación, Ciencia y Tecnología

Año VII. Vol. VII. N¹2. Enero - Junio. 2021

Hecho el depósito de ley: pp201602FA4721

ISSN-L: 2542-3029; ISSN: 2610-802X

Universidad Nacional Experimental Francisco de Miranda (UNEFM). Santa Ana de Coro. Venezuela

Alexandra Kimberly Tamay-Chimborazo; Juan Carlos Erazo-Álvarez; Cecilia Ivonne Narváez-Zurita; Cristián Andrés Erazo-Álvarez

adaptado a sus expectativas, servicios de calidad. Cuanto mayor sea esta habituación, mayor será la calidad del servicio y, por lo tanto, su satisfacción; esta última es el estado final que se alcanza cuando hay una confirmación o disconfirmación positiva entre las expectativas y percepciones del desempeño del servicio, es decir, la calidad es el primer paso y que precede a la satisfacción del comprador del servicio, que es el impacto de la calidad en los servicios. Se considera además una definición de calidad como un sistema eficiente para atraer, satisfacer, exceder y retener a todos los internos y externos clientes, efectuando las cosas a la disposición del cliente (Morillo \& Morillo, 2016).

Servicio al comprador es el diagnóstico que debería ejecutarse en la organización constantemente en delante de las necesidades y gustos del comprador debido a que este es, quien provoca que la organización gane posicionamiento". Estos esfuerzos tienen por objeto lograr una superioridad ante los usuarios y en el mercado y asegurar que el cliente se sienta satisfecho con el apoyo a largo plazo del servicio. Las organizaciones deben lograr un equilibrio entre lo que llaman un paquete sustancial de servicios y el componente de lograr el bienestar. Esto significa que el servicio al cliente es el resultado de la mezcla de ambos. Sin embargo, es necesario proporcionar un alto nivel de calidad de servicio ya que es pobre y el comprador sólo puede tolerarlo durante un período limitado de tiempo, corriendo el riesgo de perder al cliente (García, 2016).

La honestidad puede precisarse como la muestra de responsabilidad del cliente con la futura compra de productos de la propia organización o marca, principalmente como consecuencia de un episodio exitoso, estando determinado por un factor de actitud y comportamiento. La lealtad de actitud está relacionada con la propensión del cliente a comprometerse con la organización y no puede reducirse únicamente a ver un comportamiento de recompra, ejemplificando un impacto positivo de boca en boca que también refleje la reacción. Como el grado de satisfacción percibido con la recuperación del servicio reduce la propensión a cambiar de proveedor, así en este entorno tenemos 
la posibilidad de asumir que aumenta la posibilidad de recompra o la lealtad de comportamiento (Cambra-Fierro, et al., 2011).

\section{Implantación de la marca en el público objetivo}

Crear una memoria colectiva de la marca depende de varios factores, una de las más gravitantes son las campañas de difusión a través de medios convencionales y tecnológicos, especialmente las redes sociales que, en la actualidad, son las más usadas; custodiada de la producción están las promociones, mismas que sirven como enganche para captar la atención de nuevos clientes, además de mantener la fidelidad de los clientes cautivos.

Las empresas pueden posesionarse de dos maneras: el primero, el posicionamiento deseado, es el lugar que se desea ocupar por parte de la marca en la imaginación del cliente, en el tema de que la empresa quiera ofrecer bienes o servicios de fácil acceso económico, se deben ofrecer descuentos y promociones, sin embargo, si se pretende vender status, la oferta será de precios altos y calidades superiores a la media; el segundo, el posicionamiento percibido, es cuando la empresa determinar si su estrategia de marketing ha cumplido con el cometido planteado, de no ser así, se debe crear estrategias para que los clientes entiendan el mensaje de la empresa correctamente (Silva, et al., 2014).

\section{MÉTODO}

El alcance metodológico empleado ha sido descriptivo transversal no experimental, empleándose la encuesta y cuestionario online de 12 ítems y cinco alternativas de respuestas, siendo validado por juicio de expertos y fiabilidad Alfa de Cronbach de 0,87 siendo confiable, esto con la finalidad de recopilar información en una muestra poblacional de 34 personas al azar, en edades comprendidas entre los 18 y 65 años de 
CIENCIAMATRIA

Revista Interdisciplinaria de Humanidades, Educación, Ciencia y Tecnología

Año VII. Vol. VII. N¹2. Enero - Junio. 2021

Hecho el depósito de ley: pp201602FA4721

ISSN-L: 2542-3029; ISSN: 2610-802X

Universidad Nacional Experimental Francisco de Miranda (UNEFM). Santa Ana de Coro. Venezuela

Alexandra Kimberly Tamay-Chimborazo; Juan Carlos Erazo-Álvarez; Cecilia Ivonne Narváez-Zurita;

Cristián Andrés Erazo-Álvarez

edad, considerados potenciales usuarios del hotel objeto de estudio, de ese modo, se procedió a estructurar los resultados en base a la estadística descriptiva.

\section{RESULTADOS}

Los resultados de la presenta investigación son presentados, a partir de la información obtenida de la encuesta aplicada:

Se definió que el $53 \%$ de los encuestados, hace uso de instalaciones de alojamiento con una frecuencia mensual, el $9 \%$ quincenal y el $18 \%$ semanal.

Con respecto a las condiciones que consideran importantes los clientes con respecto a su decisión de uso se obtuvo que un $44 \%$ considera que la limpieza es el principal factor, el $20 \%$ toma en cuenta el precio y el $18 \%$ la atención y ubicación de las instalaciones.

De la muestra de población encuestada, se pudo obtener que el $97 \%$ considera que el motel debería aplicar estrategias de marketing experiencial, el 3\% considera que no es necesario, este resultado indica que los usuarios desean vivir experiencias sensoriales que impulsan al motel a trabajar en el desarrollo de estrategias emocionales y de estímulos a los visitantes.

\section{PROPUESTA}

En el análisis de las características que posee el marketing experiencial, se establece que se puede generar el reconocimiento de la marca Motel San Valentín en la conciencia colectiva del sector potencial de clientes.

La propuesta del marketing experiencial como estrategia de generación de ingresos dentro del Motel San Valentín, establece un esquema de cuatro etapas secuenciales especificadas a continuación: 
Revista Interdisciplinaria de Humanidades, Educación, Ciencia y Tecnología

Año VII. Vol. VII. N¹2. Enero - Junio. 2021

Hecho el depósito de ley: pp201602FA4721

ISSN-L: 2542-3029; ISSN: 2610-802X

Universidad Nacional Experimental Francisco de Miranda (UNEFM). Santa Ana de Coro. Venezuela

Alexandra Kimberly Tamay-Chimborazo; Juan Carlos Erazo-Álvarez; Cecilia Ivonne Narváez-Zurita; Cristián Andrés Erazo-Álvarez

\section{Esquema de marketing tradicional}

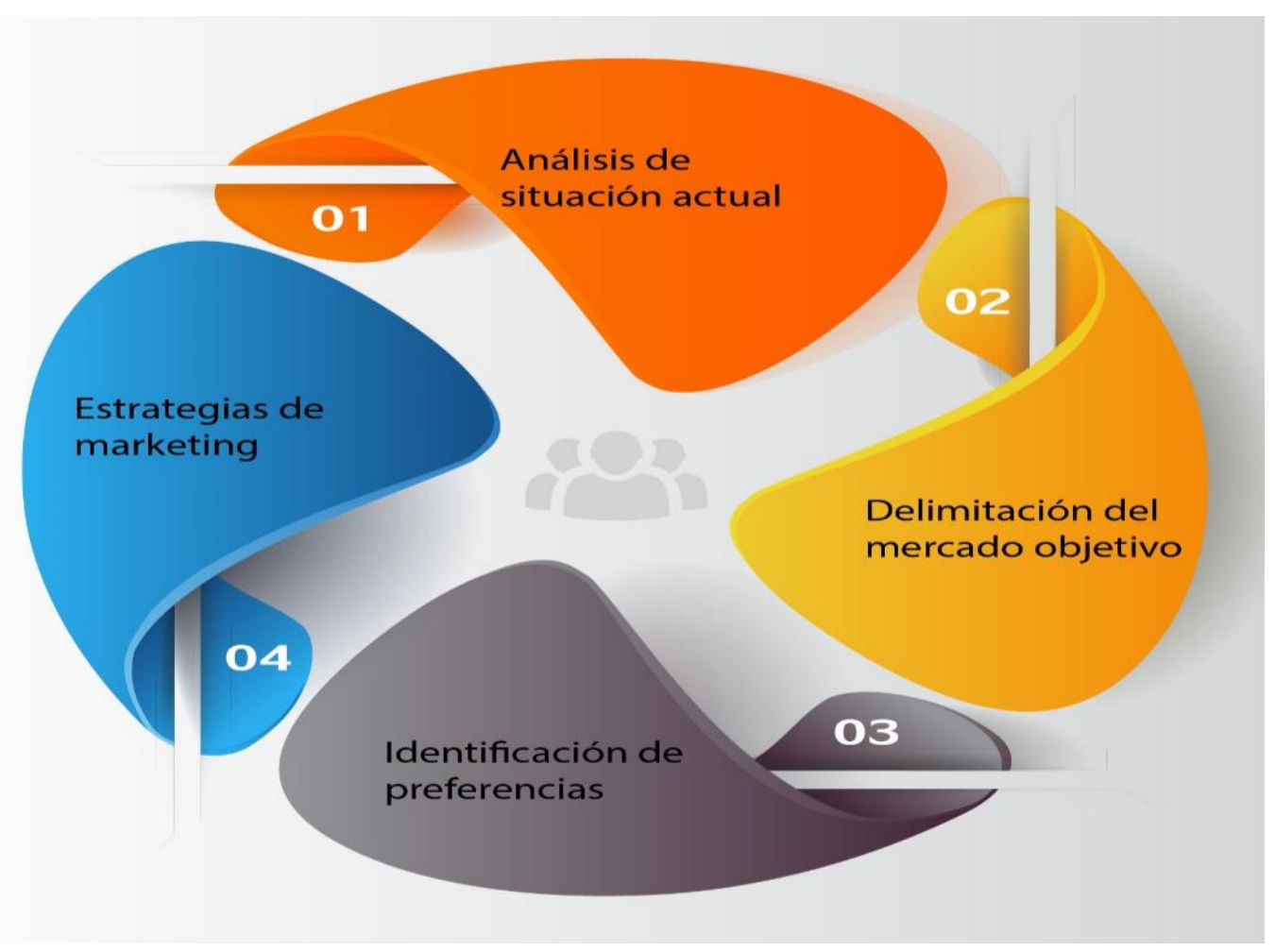

Figura 2. Esquema de la propuesta de marketing experiencial como estrategia de generación de ingresos en el Motel San Valentín.

Fuente: Elaboración propia.

\section{Análisis de situación actual}

Para tener una idea del estado inicial de la empresa, es importante un diagnóstico el cual permita marcar la estrategia y actividades a seguir para conseguir los objetivos planteados.

En la matriz FODA elaborada a continuación, se establecen los factores internos y externos de análisis, asignados pesos y calificaciones que nos ayudaran a determinar el estado de la empresa. 


\section{CIENCIAMATRIA}

Revista Interdisciplinaria de Humanidades, Educación, Ciencia y Tecnología

Año VII. Vol. VII. N¹2. Enero - Junio. 2021

Hecho el depósito de ley: pp201602FA4721

ISSN-L: 2542-3029; ISSN: 2610-802X

Universidad Nacional Experimental Francisco de Miranda (UNEFM). Santa Ana de Coro. Venezuela

Alexandra Kimberly Tamay-Chimborazo; Juan Carlos Erazo-Álvarez; Cecilia Ivonne Narváez-Zurita; Cristián Andrés Erazo-Álvarez

Para la elaboración de la matriz se debe analizar factores desde el punto de la perspectiva interna: tecnología, recursos humanos, financiación, costos de producción, control, entre otros; cuando se analiza el entorno se toma en cuenta los factores: económico, social, cultural, demográfico, ambiental, político, jurídico, tecnológico, competitivo, por nombras algunos. El peso se asigna de 0,01 a 1,00, en función del grado de influencia en el éxito de la idea; para asignar las calificaciones, en el análisis interno se toma en cuenta la posición estratégica de la empresa frente a las fortalezas y debilidades, dando los siguientes valores: 1.- Más grande debilidad, 2.- Menor debilidad, 3.- Menor fuerza y 4.Más grande fuerza, para el estudio externo se toma presente el nivel de respuesta de la táctica de hoy al elemento, aquí se califica según: 1 - Baja, 2 - Media, 3 - Superior a la media y 4 - Alta; el peso ponderado es el resultado de multiplicar el peso x calificación. Como resultado de la matriz, se obtiene un gráfico que nos muestra la situación actual de la empresa, así mismo las estrategias que se deben formular.

Tabla 2.

Análisis FODA.

\begin{tabular}{|l|r|r|r|}
\hline \multicolumn{1}{|c|}{ FACTORES INTERNOS } & PESO & CALIFICACIÓN & PESO PONDERADO \\
\hline FORTALEZAS & & & \\
\hline Instalaciones de calidad & 0,20 & 3 & 0,60 \\
\hline Experiencia en servicio de alojamiento temporal & 0,10 & 3 & 0,30 \\
\hline Cumplimiento de normas sanitarias vigentes & 0,10 & 3 & 0,30 \\
\hline Buena ubicación para acceso a clientes & 0,20 & 4 & 0,80 \\
\hline DEBILIDADES & & & 0,20 \\
\hline Habitaciones sin decoración creativa & 0,20 & 1 & 0,10 \\
\hline No se cuenta con publicidad en redes sociales & 0,10 & 1 & 0,10 \\
\hline Aplicación del marketing tradicional & 0,05 & 2 & 0,10 \\
\hline Deficiente capacitación en servicio al cliente & 0,05 & 2 & $\mathbf{2 , 5 0}$ \\
\hline TOTAL & $\mathbf{1 , 0 0}$ & & \\
\hline
\end{tabular}




\section{CIENCIAMATRIA}

Revista Interdisciplinaria de Humanidades, Educación, Ciencia y Tecnología

Año VII. Vol. VII. N¹2. Enero - Junio. 2021

Hecho el depósito de ley: pp201602FA4721

ISSN-L: 2542-3029; ISSN: 2610-802X

Universidad Nacional Experimental Francisco de Miranda (UNEFM). Santa Ana de Coro. Venezuela

Alexandra Kimberly Tamay-Chimborazo; Juan Carlos Erazo-Álvarez; Cecilia Ivonne Narváez-Zurita; Cristián Andrés Erazo-Álvarez

\begin{tabular}{|c|c|c|c|}
\hline FACTORES EXTERNOS & PESO & CALIFICACIÓN & $\begin{array}{c}\text { PESO } \\
\text { PONDERADO }\end{array}$ \\
\hline \multicolumn{4}{|l|}{ OPORTUNIDADES } \\
\hline $\begin{array}{l}\text { Industria de construcción con precios accesibles } \\
\text { para remodelaciones }\end{array}$ & 0,10 & 2 & 0,20 \\
\hline Las personas están volviendo a su vida normal & 0,20 & 2 & 0,40 \\
\hline $\begin{array}{l}\text { Clientes buscan alternativas llamativas para el uso } \\
\text { de moteles }\end{array}$ & 0,10 & 2 & 0,20 \\
\hline $\begin{array}{l}\text { La gente utiliza medios electrónicos para tomar } \\
\text { decisiones de compra }\end{array}$ & 0,15 & 1 & 0,15 \\
\hline \multicolumn{4}{|l|}{ AMENAZAS } \\
\hline Rebrote de pandemia & 0,20 & 2 & 0,40 \\
\hline Falta de servicio de taxis para clientes & 0,10 & 3 & 0,30 \\
\hline Leyes y prohibiciones & 0,05 & 2 & 0,10 \\
\hline Inconformidad por moradores del sector & 0,10 & 3 & 0,30 \\
\hline TOTAL & 1,00 & & 2,05 \\
\hline
\end{tabular}

Fuente: Elaboración propia.

\section{Situación inicial FODA Motel San Valentín}

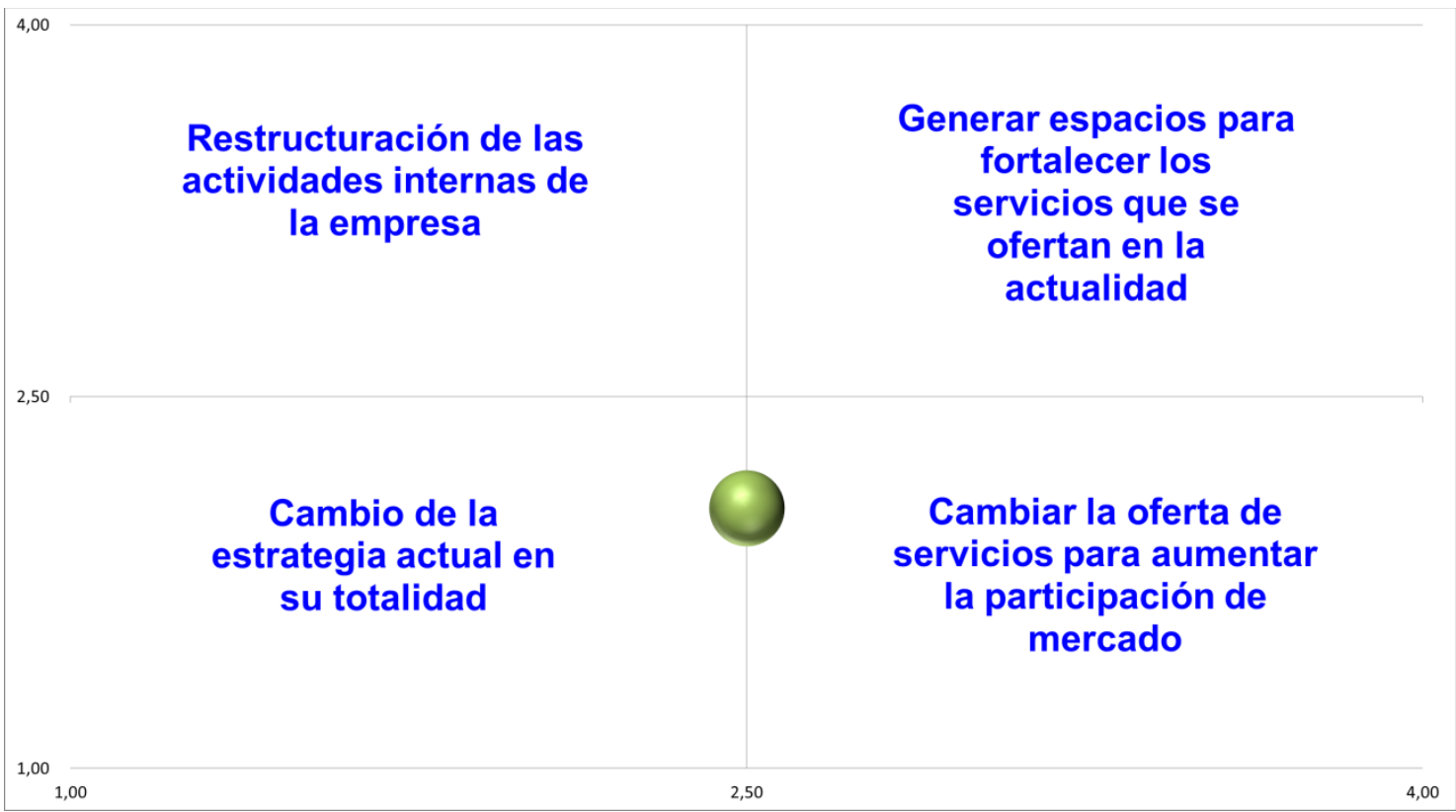

Figura 3. Situación inicial FODA Motel San Valentín.

Fuente: Elaboración propia. 


\section{CIENCIAMATRIA}

Revista Interdisciplinaria de Humanidades, Educación, Ciencia y Tecnología

Año VII. Vol. VII. N¹2. Enero - Junio. 2021

Hecho el depósito de ley: pp201602FA4721

ISSN-L: 2542-3029; ISSN: 2610-802X

Universidad Nacional Experimental Francisco de Miranda (UNEFM). Santa Ana de Coro. Venezuela

Alexandra Kimberly Tamay-Chimborazo; Juan Carlos Erazo-Álvarez; Cecilia Ivonne Narváez-Zurita; Cristián Andrés Erazo-Álvarez

\section{Delimitación del mercado objetivo}

Como parte la investigación es necesaria para identificar el mercado efectivo a quienes está dirigido las propuestas de marketing experiencial, se realiza la observación directa de los clientes que acuden al motel y se determinan sus características, con esta observación se puede plasmar un prototipo de cliente y adecuar dichas estrategias hacia su estilo de vida y preferencias.

Tabla 4.

Delimitación de mercado objetivo.

\begin{tabular}{llccc}
\hline \multicolumn{1}{c}{$\begin{array}{c}\text { Variable de } \\
\text { segmentación }\end{array}$} & \multicolumn{1}{c}{$\begin{array}{c}\text { Características } \\
\text { observadas }\end{array}$} & Datos & $\begin{array}{c}\text { Fuente de } \\
\text { información }\end{array}$ & Resultados \\
\hline Geográfica & Cuenca-Azogues & & INEC & \\
Edades & $18-65$ años & $\begin{array}{c}342.284 \\
\text { personas }\end{array}$ & INEC & 85.571 \\
Nivel socioeconómico & B y C & $25 \%$ & UPS & 25.671 \\
Estilo de vida & $\begin{array}{l}\text { Fiestas, deportistas, } \\
\text { oficinistas } \\
\text { casados, solteros, } \\
\text { amigos }\end{array}$ & $30 \%$ & $5 \%$ & $\begin{array}{c}\text { Estimado Motel San } \\
\text { Valentín }\end{array}$ \\
$\begin{array}{l}\text { Número de parejas } \\
\text { potenciales }\end{array}$ & & & 642 \\
\hline
\end{tabular}

Fuente: Elaboración propia.

Con la observación realizada se pudo determinar que el mercado que consume de forma regular los servicios de hospedaje temporal son: parejas de nivel socio-económico medioalto, casados, solteros o divorciados, viven en la ciudad, beben y fuman, en su mayoría, desean total reserva al adquirir los servicios. 
CIENCIAMATRIA

Revista Interdisciplinaria de Humanidades, Educación, Ciencia y Tecnología

Año VII. Vol. VII. N¹2. Enero - Junio. 2021

Hecho el depósito de ley: pp201602FA4721

ISSN-L: 2542-3029; ISSN: 2610-802X

Universidad Nacional Experimental Francisco de Miranda (UNEFM). Santa Ana de Coro. Venezuela

Alexandra Kimberly Tamay-Chimborazo; Juan Carlos Erazo-Álvarez; Cecilia Ivonne Narváez-Zurita; Cristián Andrés Erazo-Álvarez

\section{Identificación de preferencias experienciales}

Es importante identificar de forma permanente las tendencias para consumir de los clientes, para esto se utiliza un método de levantamiento de información por medio de encuestas anónimas, se utiliza un método cualitativo que puede ser procesado estadísticamente, utilizando criterios de medición de alto, medio y bajo, además de los rangos de edad de los usuarios del servicio de alojamiento:

\section{Tabla 5.}

Identificación de preferencias de los usuarios.

\begin{tabular}{|c|c|c|c|c|c|}
\hline \multirow{2}{*}{ Categorías de medición } & \multirow{2}{*}{ Descripción } & \multicolumn{3}{|c|}{ Percepción } & \multirow{2}{*}{ Rango de edad } \\
\hline & & Alto & Medio & Bajo & \\
\hline Emocional & $\begin{array}{l}\text { Emociones que transmite el } \\
\text { ambiente a la pareja }\end{array}$ & & & & \\
\hline \multirow{5}{*}{ Sensorial } & $\begin{array}{l}\text { Temperatura de la } \\
\text { habitación }\end{array}$ & & & & \\
\hline & Aromas & & & & \\
\hline & Alimentos y cocteles & & & & \\
\hline & Música ambiental & & & & \\
\hline & $\begin{array}{l}\text { Ambientación de la } \\
\text { habitación (Temas) }\end{array}$ & & & & \\
\hline \multirow{3}{*}{ Comunicacional } & $\begin{array}{l}\text { Retroalimentación entre } \\
\text { usuarios y personal del } \\
\text { motel }\end{array}$ & & & & \\
\hline & Publicidad en redes sociales & & & & \\
\hline & $\begin{array}{l}\text { Recomendaciones entre } \\
\text { clientes }\end{array}$ & & & & \\
\hline Intelectual & $\begin{array}{l}\text { Curiosidad por el uso de las } \\
\text { instalaciones temáticas }\end{array}$ & & & & \\
\hline
\end{tabular}

Fuente: Elaboración propia.

\section{Estrategias de marketing experiencial}

La investigación fue realizada mediante las encuestas, por medio electrónicos y de preferencias del consumo de los usuarios y delimitación del mercado objetivo, se procede con la propuesta de marketing experiencial que permitan al cliente conservar en su memoria lo que oferto el motel San Valentín (habitaciones temáticas, alimentación y 


\section{CIENCIAMATRIA}

Revista Interdisciplinaria de Humanidades, Educación, Ciencia y Tecnología

Año VII. Vol. VII. N¹2. Enero - Junio. 2021

Hecho el depósito de ley: pp201602FA4721

ISSN-L: 2542-3029; ISSN: 2610-802X

Universidad Nacional Experimental Francisco de Miranda (UNEFM). Santa Ana de Coro. Venezuela

Alexandra Kimberly Tamay-Chimborazo; Juan Carlos Erazo-Álvarez; Cecilia Ivonne Narváez-Zurita; Cristián Andrés Erazo-Álvarez

bebidas, decoración, juguetes sexuales, etc.), todo enfocado a aumentar la percepción sensorial de las experiencias vividas en las instalaciones, la difusión está ofertadas, se realizara con la utilización de redes sociales y medios de comunicación, cumpliendo de esta manera con el objetivo central de la investigación.

\section{Tabla 6.}

\section{Estrategias de marketing experiencial.}

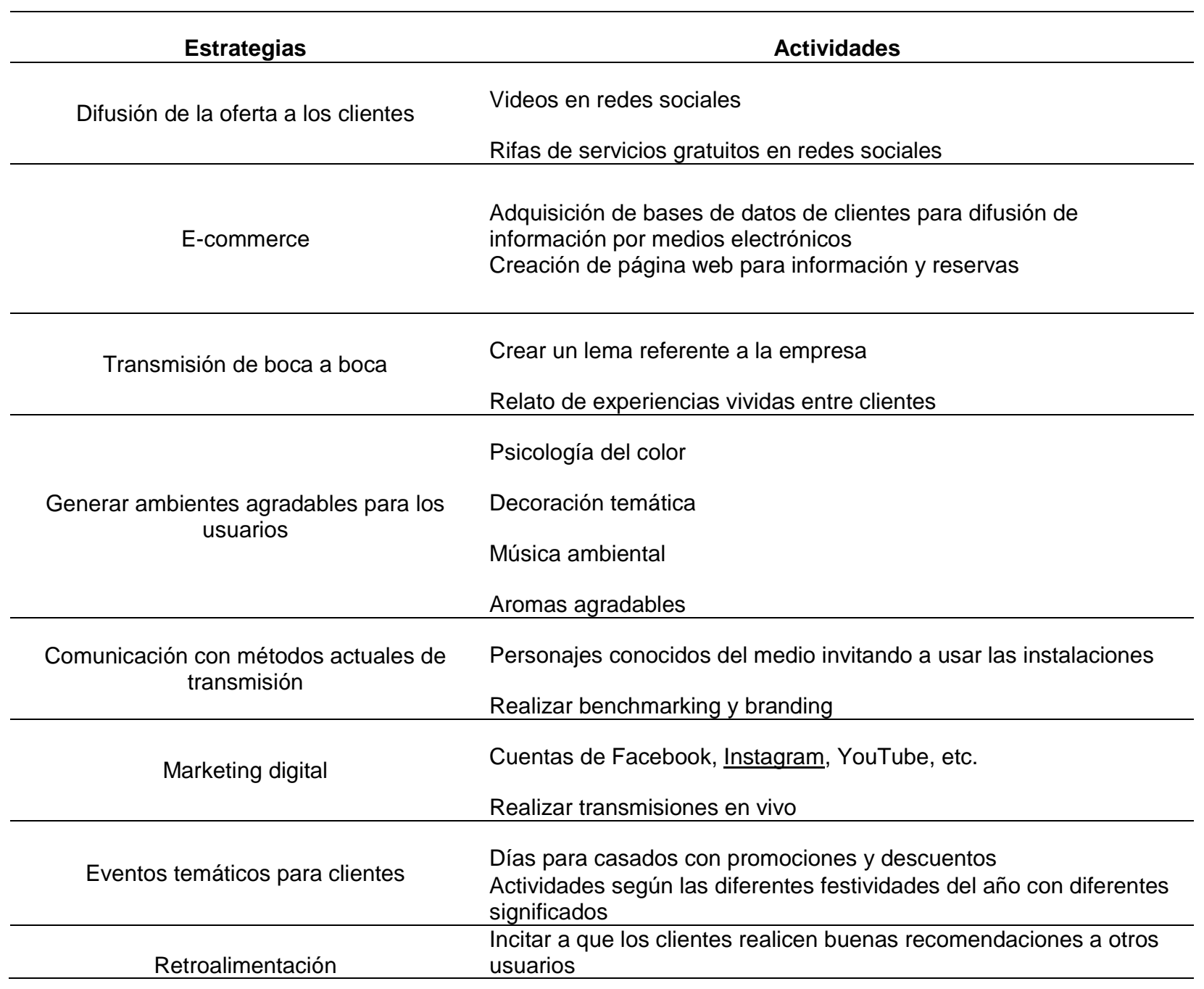

\section{Fuente: Elaboración propia.}


El enfoque del marketing experiencial a través de las indagaciones está centrado en el ámbito visual, por ese motivo a continuación detallamos algunas de las estrategias consideradas como mayor importancia para lograr los objetivos planteados:

\section{Estrategias del producto}

Motel San Valentín, está en el mercado por más de 11 años, su logotipo es un corazón, haciendo alusión a su nombre, que durante el paso de los años ha ido evolucionando, tal como se muestra en la figura siguiente:

\section{Logotipo Motel San Valentín}

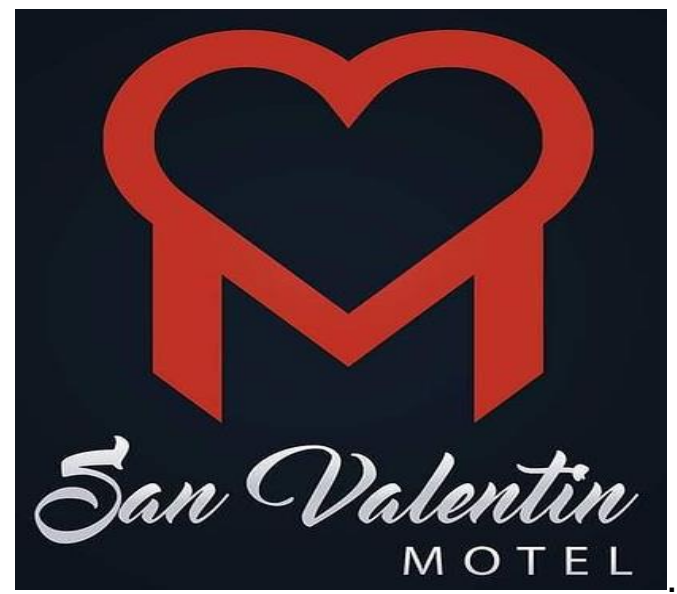

Figura 4. Logotipo Motel San Valentín.

Fuente: Motel San Valentín

\section{Estrategias de venta}

La empresa tiene su infraestructura de servicios en la ciudad de Biblián, sus ventas son directas, esto obedece a que los usuarios deben acudir a su negocio, por este motivo se plantea la posibilidad de conocer, de forma virtual, las opciones que ofrecen del negocio y a su vez, la posibilidad de hacer reservas por medio de una cuenta en la plataforma Facebook. 


\section{CIENCIAMATRIA}

Revista Interdisciplinaria de Humanidades, Educación, Ciencia y Tecnología

Año VII. Vol. VII. Nº12. Enero - Junio. 2021

Hecho el depósito de ley: pp201602FA4721

ISSN-L: 2542-3029; ISSN: 2610-802X

Universidad Nacional Experimental Francisco de Miranda (UNEFM). Santa Ana de Coro. Venezuela

Alexandra Kimberly Tamay-Chimborazo; Juan Carlos Erazo-Álvarez; Cecilia Ivonne Narváez-Zurita; Cristián Andrés Erazo-Álvarez

\section{Cuenta de Facebook Motel San Valentín}

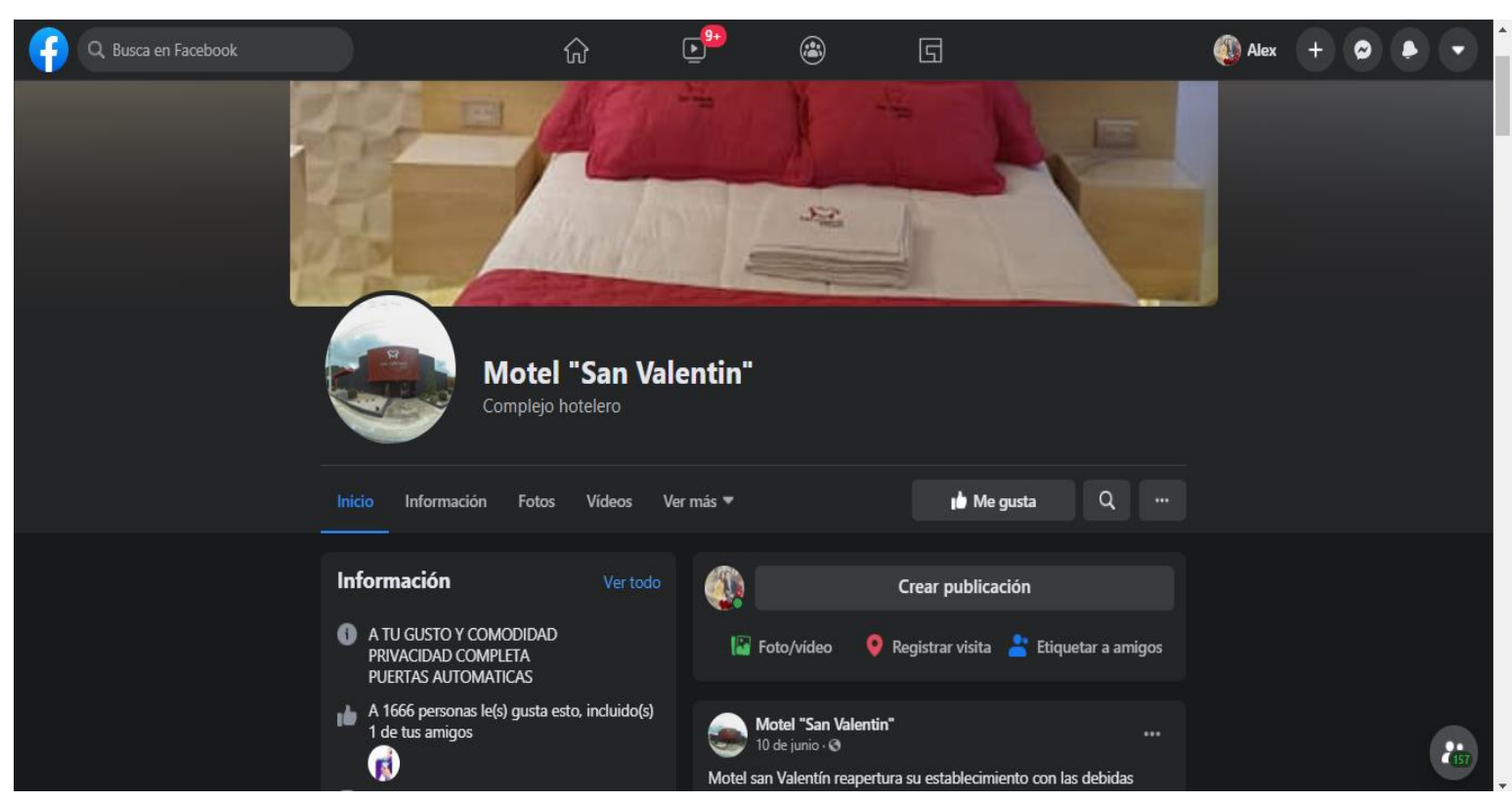

Figura 5. Cuenta Motel San Valentín en Facebook.

Fuente: https://cutt.ly/AkQl2SR 


\section{CIENCIAMATRIA}

Revista Interdisciplinaria de Humanidades, Educación, Ciencia y Tecnología

Año VII. Vol. VII. N¹2. Enero - Junio. 2021

Hecho el depósito de ley: pp201602FA4721

ISSN-L: 2542-3029; ISSN: 2610-802X

Universidad Nacional Experimental Francisco de Miranda (UNEFM). Santa Ana de Coro. Venezuela

Alexandra Kimberly Tamay-Chimborazo; Juan Carlos Erazo-Álvarez; Cecilia Ivonne Narváez-Zurita; Cristián Andrés Erazo-Álvarez

Se expone la construcción de contenido para la plataforma Instagram con varios temas de interés, dirigido para el mercado objetivo.

\section{Cuenta de Instagram Motel San Valentín}

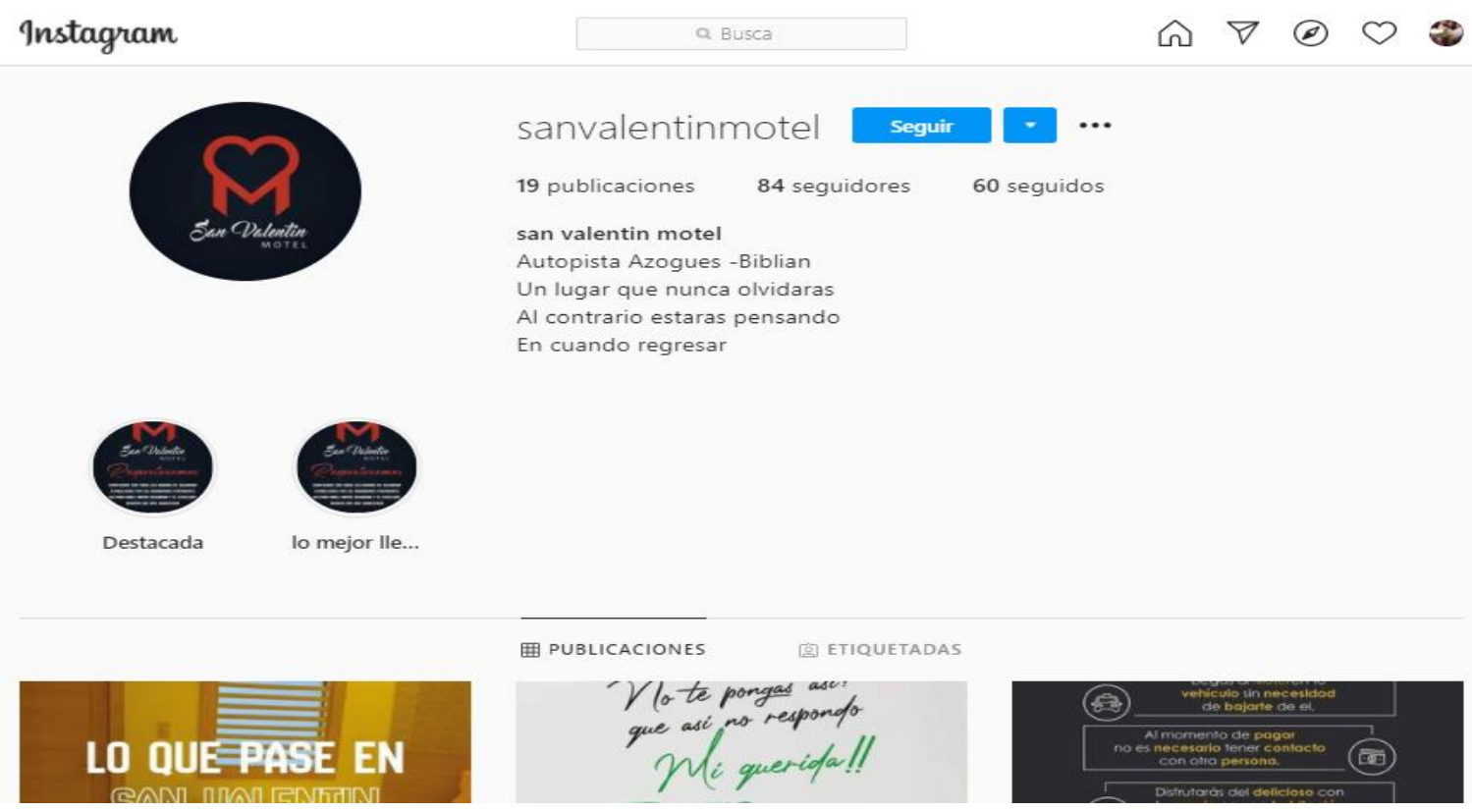

Figura 6. Cuenta Motel San Valentín en Instagram.

Fuente: https://cutt.ly/PkQ173a

\section{CONCLUSIONES}

La iniciativa cumple el propósito postulado en la investigación de realizar actividades de marketing experiencial como táctica de generación de ingresos en el Motel San Valentín, la ejecución de la iniciativa en cuestión ayuda a la dirección de la organización en la toma de decisiones, tanto económicas como administrativas.

En el desarrollo de la investigación se sugiere que el marketing experiencial busca generar una conexión emocional entre la marca y el comprador que los lleve a convertirse en fieles entusiastas de la marca, dispuestos a compartir su experiencia con otras 


\begin{abstract}
CIENCIAMATRIA
Revista Interdisciplinaria de Humanidades, Educación, Ciencia y Tecnología

Año VII. Vol. VII. N¹2. Enero - Junio. 2021

Hecho el depósito de ley: pp201602FA4721

ISSN-L: 2542-3029; ISSN: 2610-802X

Universidad Nacional Experimental Francisco de Miranda (UNEFM). Santa Ana de Coro. Venezuela

Alexandra Kimberly Tamay-Chimborazo; Juan Carlos Erazo-Álvarez; Cecilia Ivonne Narváez-Zurita; Cristián Andrés Erazo-Álvarez
\end{abstract}

personas dentro del motel, para lo cual se utilizarán diversas tácticas apelando a todos los sentidos probables que provoquen una inmersión emocional del comprador.

En este momento, el uso de Internet y las redes sociales, permiten establecer una interacción más estrecha entre el comprador y la empresa que debe ser utilizada para el desarrollo del contenido de la comunicación, de manera que el comprador satisfaga todas las necesidades de información relativas al comercio, decida adquirir los servicios prestados por la organización.

\title{
REFERENCIAS CONSULTADAS
}

Barrios, M. (2012). Marketing de la Experiencia: principales conceptos [Experience Marketing: main concepts]. Palermo Business Review, № 7, 67-89. Obtenido de https://n9.cl/1i73

Bernal, A., \& Galvis, J. (2008). El marketing sensorial como herramienta para el fortalecimiento de la imagen corporativa [Sensory marketing as a tool for strengthening corporate image]. Obtenido de http://hdl.handle.net/10554/5134

Cadavid-Gómez, H. (2004). Marketing de emociones. La forma para lograr fidelidad de los clientes. Semestre Económico, 7(13),203-211.

Calle-Calderón, K., Erazo-Álvarez, J., \& Narváez-Zurita, C. (2020). Marketing digital y estrategias online en el sector de fabricación de muebles de madera. Revista Arbitrada Interdisciplinaria Koinonía, 5(10), 339-369. http://dx.doi.org/10.35381/r.k.v5i10.698

Cambra-Fierro, J, \& Ruiz-Benítez, R, \& Berbel-Pineda, J, \& Vázquez-Carrasco, R. (2011). Podemos fidelizar clientes inicialmente insatisfechos. Revista de Ciencias Sociales (Ve), XVII(4),643-657.

Carbache-Mora, C, Ureta-Ureta, S, \& Nevarez-Vera, J. (2019). Aporte del storytelling para la creación del marketing emocional en empresa de agua purificada de bahía de Caráquez Ecuador

2019. Comuni@cción, 10(2), $140-$ 150. https://dx.doi.org/10.33595/2226-1478.10.2.386 


\section{CIENCIAMATRIA}

Revista Interdisciplinaria de Humanidades, Educación, Ciencia y Tecnología

Año VII. Vol. VII. N¹2. Enero - Junio. 2021

Hecho el depósito de ley: pp201602FA4721

ISSN-L: 2542-3029; ISSN: 2610-802X

Universidad Nacional Experimental Francisco de Miranda (UNEFM). Santa Ana de Coro. Venezuela

Alexandra Kimberly Tamay-Chimborazo; Juan Carlos Erazo-Álvarez; Cecilia Ivonne Narváez-Zurita; Cristián Andrés Erazo-Álvarez

Caridad, M, \& Castellano, M, \& Hernández, M. (2015). Marketing experiencial para la promoción del proceso educativo a nivel de pregrado: un estudio de dos universidades privadas. Revista de la Facultad de Ciencias Económicas: Investigación y Reflexión, XXIII(2),81-95.

Cepeda-Palacio, S. (2014). Alcances actuales del concepto de marca. Un estudio comparativo, en la historia. Entramado, 10(2),128-142.

De-Garcillán-López-Rúa, M. (2015). Persuasión a través del marketing sensorial y experiencial. Opción, 31(2),463-478.

García, A. (2016). Cultura de servicio en la optimización del servicio al cliente. Telos, 18(3),381-398.

Klotler, P., \& Armstrong, G. (2008). Fundamentos de Marketing. Mexico: Pearson Education. Recuperado de https://n9.cl/7z42.

Küster, I, Vila, N, \& Canales, P. (2008) El marketing relacional y el marketing emocional. Estudios sobre Consumo 84

Lazo-Torres, N., Erazo-Álvarez, J., \& Narvaéz-Zurita, C. (2019). El Balanced Scorecard como herramienta de control interno en el sector Manufacturero. Revista Arbitrada Interdisciplinaria Koinonía, 4(1), 125-152. http://dx.doi.org/10.35381/r.k.v4i1.374

Lobera, E. (2018). El marketing experiencial y su importancia para el estudio del e-WOM [Experiential marketing and its importance for the study of e-WOM]. MarketingSocial Media, 1-2. Obtenido de https://n9.cl/jivl

Mejías-Acosta, A., Godoy Durán, E., \& Piña Padilla, R. (2018). Impacto de la calidad de los servicios sobre la satisfacción de los clientes en una empresa de mantenimiento. Revista Científica Compendium, 21(40).

Moreno, O. (2007). El marketing internacional como herramienta indispensable para una exportación efectiva. Ciencia en su PC, (2),1-10.

Morillo-Moreno, M, \& Morillo-Moreno, M. (2016). Satisfacción del usuario y calidad del servicio en alojamientos turísticos del estado Mérida, Venezuela. Revista de Ciencias Sociales (Ve), XXII(2),111-131. 


\section{CIENCIAMATRIA}

Revista Interdisciplinaria de Humanidades, Educación, Ciencia y Tecnología

Año VII. Vol. VII. N¹2. Enero - Junio. 2021

Hecho el depósito de ley: pp201602FA4721

ISSN-L: 2542-3029; ISSN: 2610-802X

Universidad Nacional Experimental Francisco de Miranda (UNEFM). Santa Ana de Coro. Venezuela

Alexandra Kimberly Tamay-Chimborazo; Juan Carlos Erazo-Álvarez; Cecilia Ivonne Narváez-Zurita; Cristián Andrés Erazo-Álvarez

Olivera-Carhuaz, E.S., \& Pulido-Capurro, V.M.. (2018). Marketing social: Su importancia en la resolución de problemas sociales. Revista Científica de la UCSA, 5(2), 2635. https://dx.doi.org/10.18004/ucsa/2409-8752/2018.005(02)026-035

Ordónez, A., \& Prieto, V. (2019). Marketing experiencial, una tendencia en auge [Experiential marketing, a growing trend]. Obtenido de https://n9.cl/02x4

Ramírez, DE., Duarte, C. \& Toro, LM. (2016). La emocionalidad en las estrategias de mercadeo: caso industria funeraria. Dimensión Empresarial, 14(1), 109-122. http://dx.doi.org/10.15665/rde.v14i1.570

Reyes, P. E., Narváez, C. I., Erazo, J. C., \& Giler, L. V. (2020). Configuración del impuesto a la patente municipal con base al ingreso de las actividades económicas. Caso: GAD Municipal de Pucará - Ecuador. Revista Espacios, 41(21), 197-211.

Salas-Canales, H.J. (2018). Neuromarketing: Explorando la mente del consumidor. Revista Científica de la UCSA, 5(2), 3644. https://dx.doi.org/10.18004/ucsa/2409-8752/2018.005(02)036-044

Schimitt, B. (2000). Marketing Experiencial [Experiential Marketing] Recuperado de https://n9.cl/ngt5.

Silva , H., Martínez , D., Giraldo , M., \& Juliao, D. (2014). Markeying Conceptos y Aplicaciones. [Marketing Concepts and Applications] Recuperado de https://n9.cl/a7zv.

Suárez del Villar, S., \& Salazar Caballero, B. (2016). El marketing emperiencial como herramienta para el fortalecimiento de la imagen corporativa de las empresas del sector comercial Colombiano. Dictamen Libre, (19), 97-109. https://doi.org/10.18041/2619-4244/dl.19.2905 